\title{
Comparação do uso tópico de cetotifeno com a olopatadina no tratamento de conjuntivites alérgicas
}

\author{
Comparison between topical use of Ketotifen and olopatadine in the treatment of allergic \\ conjunctivitis
}

\author{
Ana Luisa Höfling-Lima ${ }^{1}$ \\ Alfredo J. M. Andrade \\ Patrícia M. F. Marback $k^{2}$ \\ Michel Eid Farah ${ }^{3}$ \\ Vera Mascaro $^{4}$
}

\section{RESUMO}

Objetivo: Avaliar e comparar a eficácia e tolerância do uso tópico do fumarato de cetotifeno a $0,05 \%$ e cloridrato de olopatadina a $0,1 \%$ no tratamento de pacientes com conjuntivite alérgica. Método: Foi realizado estudo clínico mascarado, randomizado comparando a eficácia, segurança e os efeitos colaterais com o uso da solução oftálmica de fumarato de cetotifeno a $0,05 \%$ e cloridrato de olopatadina a $0,1 \%$ no alívio dos sintomas e sinais em pacientes com conjuntivite alérgica. Trinta e quatro pacientes obedecendo aos critérios de inclusão do protocolo receberam um frasco com a droga mascarada e instilaram uma gota duas vezes por dia em cada olho durante 30 dias. Os sintomas e sinais dos pacientes foram avaliados em uma visita pré-tratamento e cinco com tratamento $\left(1^{\circ} \mathrm{dia}, 2^{\circ}\right.$ dia, $7^{\circ}$ dia, $14^{\circ}$ dia e $30^{\circ}$ dia). Resultados: A gravidade da conjuntivite alérgica foi semelhante nos dois grupos do estudo. Tanto cetotifeno como a olopatadina foram equivalentes e eficazes na diminuição dos sintomas de prurido, ardor e lacrimejamento. Quanto aos sinais, a hiperemia em conjuntiva bulbar foi atenuada nos dois grupos. Na avaliação das reações adversas observou-se ardor após a administração de ambos colírios e a ocorrência de prurido no grupo do cetotifeno. Não foi observada nenhuma reação de hipersensibilidade das drogas estudadas. Conclusões: Este estudo evidencia que a solução oftálmica de fumarato de cetotifeno a $0,05 \%$ e o cloridrato de olopatadina a $0,1 \%$ quando instilados duas vezes ao dia durante 30 dias, são eficazes e seguros no alívio dos principais sintomas das conjuntivites alérgicas.

Descritores: Cetotifeno/uso terapêutico; Dibenzoxepinas/uso terapêutico; Soluções oftálmicas; Conjuntivite alérgica/quimioterapia

INTRODUÇÃO

As alergias oculares são doenças freqüentes e representam uma importante porcentagem das consultas em clínicas oftalmológicas. Os pacientes alérgicos raramente têm comprometimento da visão, porém apresentam, em alguns casos, intenso desconforto ${ }^{(1)}$.

A manifestação da alergia ocular ocorre com alterações nas conjuntivas oculares e nas pálpebras onde os mastócitos destes tecidos, quando ativados, liberam mediadores da inflamação pré-formados em seus grânulos e mediadores recém sintetizados de suas membranas celulares através da cascata do ácido aracdônico causando as manifestações clínicas da alergia ${ }^{(2-4)}$.

Os principais sintomas de alergia ocular são: prurido e a hiperemia conjuntival, podendo existir outros sintomas como lacrimejamento, secre- 
ção mucóide, fotofobia, sensação de corpo estranho. Grande variação do quadro clínico é observado para paciente em períodos diferentes ${ }^{(5)}$.

No exame citológico da conjuntiva em pacientes com alergia conjuntival observa-se eosinófilos com freqüência que varia de $20-80 \%$ dependendo do momento em que a amostra for colhida, do tempo de manifestação alérgica e da freqüência de repetição do exame citológico ${ }^{(6-7)}$.

Das drogas usadas para tratamento tópico destacamos dois medicamentos recentemente disponibilizados no Brasil que são a olopatadina ${ }^{(4,8)}$ e o cetotifeno ${ }^{(9-10)}$ com ação de bloqueio nos receptores $\mathrm{H}_{1}$ e estabilização dos mastócitos. $\mathrm{O}$ cetotifeno é uma medicação já usada por via oral e agora disponibilizada para uso tópico, possui propriedade de antihistamínico $\mathrm{H}_{1}$ de $2^{\mathrm{a}}$ geração e antianafiláticas, agindo em várias vias da inflamação, como nos mediadores presentes nos neutrófilos, nas proteases dos mastócitos, e inibe os mediadores da reação lenta da anafilaxia ${ }^{(10-1)}$. Esta droga tem descrito baixa atividade antiserotoninérgica e anticolinérgi$\mathrm{ca}^{(9)}$. Outras propriedades pouco conhecidas seria a de inibição do fator da atividade plaquetária e redução nas funções dos eosinófilos e sua viabilidade ${ }^{(11-12)}$. Ambas drogas estão liberadas para uso no Brasil.

A medicação antialérgica ideal deveria ser única (monoterapia) e com poucas instilações por dia, apresentar rapidez no início da ação, com longa duração do efeito sobre os sintomas e sinais e não levar ao aparecimento de reações adversas, e também não desenvolver taquifilaxia ou hipersensibilidade, sem interação medicamentosa e de baixo custo.

OBJETIVO

Este estudo tem como objetivo verificar e comparar a eficácia, segurança e as reações adversas do tratamento tópico da conjuntivite alérgica com cetotifeno e olopatadina.

\section{MÉTODOS}

Foi realizado um estudo clínico mascarado e randomizado, com pacientes portadores de conjuntivite alérgica, no período de 01 de fevereiro a 30 de junho de 1999. Após a avaliação dos pacientes e obedecendo aos critérios de inclusão e exclusão foi feita a comunicação da possibilidade de participação no estudo. Na seqüência foi esclarecido a cada paciente o conteúdo do protocolo, com a leitura e assinatura do termo de consentimento.

Critérios de inclusão: pacientes em qualquer faixa etária, com diagnóstico clínico de conjuntivite alérgica, do sexo masculino e feminino e que permitissem o exame oftalmológico adequado.

Critérios de exclusão: 1) pacientes portadores de conjuntivite alérgica e que necessitassem de tratamento sistêmico ou tópico com corticosteróide; 2) portadores de hipersensibilidade conhecida a qualquer um dos componentes da droga do estudo; 3) portadores de glaucoma de ângulo fechado; 4) outras enfermidades oculares que possam interferir nos resultados; 5) uso concomitante com outra medicação ocular; 6) mulheres grávidas ou lactantes; 7) uso de lentes de contato durante o período do estudo; 8) enfermidades sistêmicas e não controladas ex.: Diabetes e Hipertensão Arterial; 9) que não tivessem o tempo mínimo de cinco dias de interrupção do uso de qualquer outra medicação tópica; 10) uso via oral de hipoglicemiantes orais, medicamentos depressores do sistema nervoso central e hábito de ingestão de bebidas alcoólicas.

De cada paciente os sinais e sintomas somente de um olho foi computado para este estudo e a escolha do olho para análise por sorteio "cara ou coroa", considerou-se olho direito como cara e olho esquerdo como coroa. A introdução do tratamento foi randomizada previamente e os frascos com as medicações tópicas foram mascarados pelo Laboratório Ophthalmos que obteve a olopatadina $0,1 \%\left(\mathrm{Patanol}^{\circledR}\right)$ diretamente em farmácias locais e o cetotifeno $0,05 \%$ fornecido pelo fabricante (CIBA Vision). Nos Quadros 1 e 2 estão

\begin{tabular}{|c|c|}
\hline \multicolumn{2}{|r|}{ Características } \\
\hline Nome Comercial & $\begin{array}{l}\text { Solução Oftálmica de } \\
\text { Fumarato de Cetotifeno } 0,05 \%\end{array}$ \\
\hline Componente ativos & $\begin{array}{l}0,69 \mathrm{mg} \text { Fumarato de Cetotifeno } \\
\text { equivalente a } 0,5 \mathrm{mg} \text { de Cetotifeno }\end{array}$ \\
\hline Componentes inativos & $\begin{array}{l}\text { Glicerol, Hidróxido de Sódio/Ácido } \\
\text { Clorídico (para ajuste de } \mathrm{pH} \text { ), } \\
\text { Água destilada }\end{array}$ \\
\hline Preservante & Cloreto de Benzalcônio 0,01\% \\
\hline Cor / aspecto da solução & Branca/cristalino \\
\hline Solubilidade & Hidrossolúvel \\
\hline Peso molecular & $425,50 \mathrm{Mol}$ \\
\hline $\mathrm{PH}$ & $4,4-5,8$ \\
\hline Osmolalidade da solução & $210-300 \mathrm{mOsmol} / \mathrm{Kg}$ \\
\hline
\end{tabular}

\begin{tabular}{|c|c|}
\hline & aracterísticas \\
\hline Nome Comercial & $\begin{array}{l}\text { Solução Oftálmica de } \\
\text { Cloridrato de Olopatadina } 0,1 \%\end{array}$ \\
\hline Componente ativos & $\begin{array}{l}1,11 \mathrm{mg} \text { Cloridrato de Olopatadina } \\
\text { equivalente a } 1 \mathrm{mg} \text { de Olopatadina }\end{array}$ \\
\hline Componentes inativos & $\begin{array}{l}\text { Fosfato de Sódio dibásico, Cloreto de } \\
\text { sódio, Glicerol, Hidróxido de Sódio/ } \\
\text { Ácido Clorídico (para ajuste de } \mathrm{pH} \text { ), } \\
\text { Água destilada }\end{array}$ \\
\hline Preservante & Cloreto de Benzalcônio 0,01\% \\
\hline Cor / aspecto da solução & Branca/cristalino \\
\hline Solubilidade & Hidrossolúvel \\
\hline Peso molecular & $373,88 \mathrm{Mol}$ \\
\hline $\mathrm{PH}$ & 7 \\
\hline Osmolalidade da solução & $300 \mathrm{mOsmol} / \mathrm{Kg}$ \\
\hline
\end{tabular}


descritos os componentes e propriedades físico-químicas das soluções com as drogas utilizadas neste estudo. Somente os monitores médicos entregaram aos pacientes os medicamentos.

O tratamento prescrito com as duas drogas foi de instilação no fundo de saco conjuntival de uma gota duas vezes por dia em ambos olhos, com intervalo de 12 horas, observando após 30 minutos em média da instilação a presença de algum incômodo nos seus olhos. Os pacientes foram conscientizados de que deveriam utilizar o colírio ininterruptamente por 30 dias e que o uso de qualquer outra medicação tópica ou sistêmica sem o prévio conhecimento dos médicos não seria desejável. Todas as avaliações foram realizadas por dois médicos oftalmologistas treinados previamente quanto à padronização dos critérios das avaliações e em cada item avaliado a opinião dos médicos deviam concordar, caso não seria revisto caso em separado, num total de 6 visitas, na seguinte distribuição: uma antes do início do tratamento e outra no $1^{\circ}$ dia, $2^{\circ}$ dia, $7^{\circ} \mathrm{dia}, 14^{\circ} \mathrm{dia}$ e $30^{\circ} \mathrm{dia}$. Somente os pacientes com todas as visitas realizadas, tiveram seus dados analisados no final do protocolo.

As informações do olho avaliado no protocolo foram documentados em questionários individuais para cada visita o que permitiu a análise seqüencial dos resultados. Em todas as visitas foram observados os sintomas, sinais e reações adversas em ambos olhos. Os sintomas: prurido; ardor; lacrimejamento; secreção e fotofobia; que foram graduados com base num escore de acordo com as respostas dos pacientes às perguntas feitas pelos monitores médicos, de acordo com o grau de gravidade de 0, 1, 2 e 3 (Quadro 2).

Os sinais observados foram: hiperemia conjuntival; edema da pálpebra, conjuntiva e limbo; presença e grau da gravidade das papilas na conjuntiva bulbar e grau de cicatrização na conjuntiva tarsal superior; presença e grau do pannus vascular na região límbica; presença e intensidade dos nódulos de "Trantas"; intensidade da coloração da conjuntiva bulbar com fluoresceína; presença do tipo de ceratite epitelial se difuso ou localizado. Todos os sinais foram graduados segundo a presença e grau de gravidade num escore de $0,1,2$ a 3 (Quadro 4).

A utilização deste tipo de formato nas escalas permite que a soma total dos escores de graduação para cada variável, entre o dia inicial e o $30^{\mathrm{a}}$ dia, resulte um número entre 0 e 18 .

A avaliação dos efeitos adversos foi analisada em todas as visitas sendo observados: prurido; ardor; cefaléia; olho seco; sensação de corpo estranho; alteração no paladar e presença de crises de rinite. A graduação dos efeitos colaterais seguiu os mesmos critérios utilizados para os sintomas oculares (Quadro 3).

O menisco lacrimal foi avaliado por dois testes: teste de Schirmer e o teste de tempo de ruptura do filme lacrimal, este último foi realizado com fluoresceína bastão sem a utilização de colírio anestésico prévio, sendo o bastão umedecido com soro fisiológico a $0,9 \%$. Os exames laboratoriais, com a reali-

\begin{tabular}{|c|c|c|c|c|}
\hline \multicolumn{5}{|c|}{$\begin{array}{c}\text { Quadro 3. Definição dos escores das graduações dos sintomas } \\
\text { nas conjuntivites alérgicas }\end{array}$} \\
\hline \multirow[t]{2}{*}{ Sintomas } & \multicolumn{4}{|c|}{ Graduação dos Sintomas } \\
\hline & $\begin{array}{l}\text { Ausente } \\
\text { (Grau 0) }\end{array}$ & $\begin{array}{r}\text { Leve } \\
\text { (Grau 1) }\end{array}$ & $\begin{array}{r}\text { Moderado } \\
\text { (Grau 2) }\end{array}$ & $\begin{array}{r}\text { Grave } \\
\text { (Grau 3) }\end{array}$ \\
\hline $\begin{array}{l}\text { Prurido } \\
\text { Ardor } \\
\text { Lacrimejamento } \\
\text { Secreção } \\
\text { Fotofobia }\end{array}$ & Ausente & $\begin{array}{l}\text { Apenas } \\
\text { visível }\end{array}$ & $\begin{array}{c}\text { Claramente } \\
\text { visível mas } \\
\text { não grave }\end{array}$ & $\begin{array}{r}\text { Claramente } \\
\text { visível e } \\
\text { grave }\end{array}$ \\
\hline
\end{tabular}

\begin{tabular}{|c|c|c|c|c|}
\hline \multicolumn{5}{|c|}{$\begin{array}{l}\text { Quadro 4. Definição dos escores das graduações dos sinais nas } \\
\text { conjuntivites alérgicas }\end{array}$} \\
\hline \multirow[t]{2}{*}{ Sinais } & \multicolumn{4}{|c|}{ Graduação dos Sinais } \\
\hline & $\begin{array}{l}\text { Ausente } \\
\text { (Grau 0) }\end{array}$ & $\begin{array}{l}\text { Leve } \\
\text { (Grau 1) }\end{array}$ & $\begin{array}{l}\text { Moderado } \\
\text { (Grau 2) }\end{array}$ & $\begin{array}{l}\text { Grave } \\
\text { (Grau 3) }\end{array}$ \\
\hline $\begin{array}{l}\text { Hiperemia } \\
\text { Edema pálpebra } \\
\text { Edema conjuntiva } \\
\text { Cicatrizes tarsais } \\
\text { Neovascularização } \\
\text { Nódulos de Trantas } \\
\text { Fluoresceína } \\
\text { Ceratite difusa } \\
\text { Ceratite em fenda }\end{array}$ & Ausente & $\begin{array}{l}\text { Apenas } \\
\text { visível }\end{array}$ & $\begin{array}{l}\text { Claramente } \\
\text { visível } \\
\text { mas não } \\
\text { grave }\end{array}$ & $\begin{array}{c}\text { Claramente } \\
\text { visível } \\
\text { e grave }\end{array}$ \\
\hline
\end{tabular}

zação do raspado de conjuntiva tarsal inferior em 3 lâminas e a cultura em meio sólido de ágar chocolate, analisados pelo Laboratório de Microbiologia Ocular da UNIFESP/EPM. O exame citológico da conjuntiva tarsal inferior foi realizado na visita pré-tratamento e no $30^{\circ}$ dia em ambos olhos dos pacientes do estudo, observando nas amostras a contagem de grânulos livres, eosinófilos degenerados ou íntegros e a contagem neutrófilos degenerados.

No $30^{\circ}$ dia de tratamento a medicação foi recolhida dos pacientes e os frascos ficaram armazenados no Laboratório Ophthalmos por 30 dias.

Ficou previsto no início do estudo a possibilidade de abertura do envelope individual correspondente ao número do paciente para esclarecimentos no caso de intercorrência com a informação sobre a droga de uso, caso este procedimento fosse necessário o paciente automaticamente sairia do estudo em qualquer fase do estudo. Os monitores médicos não poderiam alterar o protocolo do estudo uma vez iniciado, e os critérios éticos foram conduzidos conforme os princípios da Declaração de Helsinque.

Os testes estatísticos foram realizados pelo programa de computação SPSS $^{\circledR}$, versão 8.0 (SPSS Brasil Ltda.). Foram utilizados: teste de Friedman ${ }^{(13)}$, complementado pelo teste das comparações múltiplas ${ }^{(14)}$, quando necessário; teste do qui-quadrado ${ }^{(13)}$ foi aplicado obedecendo-se às restrições de Cochran, e neste caso quando presentes usamos o teste exato de Fisher ${ }^{(13)}$ e o teste de Mann-Whitney ${ }^{(13)}$. Para rejeição da hipótese de nulidade usamos um valor de $\mathrm{p} \leq 0,05$, simbolizado 
quando significante com um asterisco $(*)$. Todos os testes foram considerados bicaudais. As médias foram calculadas e apresentadas apenas a título de informação.

\section{RESULTADOS}

Dos quarenta pacientes com conjuntivite alérgica que foram avaliados para iniciar o estudo apenas 34 compareceram a todas as visitas, sendo assim seis pacientes foram excluídos do estudo por não terem comparecido a todas avaliações recomendadas no protocolo. A investigação dos motivos destas falhas no comparecimento esclareceu que os pacientes relataram a dificuldade de locomoção na cidade nos dias marcados, excluindo-se assim outras causas para o não comparecimento. Nenhum caso de hipersensibilidade às drogas do estudo foi observado nos pacientes avaliados.

Todos pacientes incluídos no estudo apresentaram melhora dos seus sintomas ao final do tratamento independente do grupo analisado.

A análise da eficácia das drogas do estudo foi feita com 34 pacientes que cumpriram o protocolo completo, divididos em 18 pacientes que receberam tratamento com fumarato de cetotifeno a $0,05 \%$ e 16 com tratamento tópico com cloridrato de olopatadina a $0,1 \%$. Dezenove pacientes eram do sexo feminino e 15 do sexo masculino, com idade média 27 anos (5 até 73 anos).

Entre os dois grupos foi demonstrado homogeneidade da amostra em relação à gravidade de conjuntivite alérgica, sinais e sintomas das conjuntivites (Tabela 1), pois dezoito pacientes que usaram cetotifeno a $0,05 \%, 15$ eram portadores de quadro alérgico leve e 3 com quadro moderado e dos 16 pa-

\begin{tabular}{|c|c|c|c|c|}
\hline \multicolumn{5}{|c|}{$\begin{array}{l}\text { Tabela 1. Soma dos escores das graduações do grau de gravidade } \\
\text { dos sintomas, sinais em pacientes com conjuntivite alérgica } \\
\text { tratados com colírio de cetotifeno }(N=18) \text { ou olopatadina }(N=16) \text { e } \\
\text { resultados estatísticos }\end{array}$} \\
\hline \multirow{2}{*}{\multicolumn{2}{|c|}{ Variáveis analisadas }} & \multicolumn{2}{|c|}{ Médias } & \multirow[b]{2}{*}{$\begin{array}{l}\text { Teste de } \\
\text { Mann-Whitney } \\
\text { z calc }\end{array}$} \\
\hline & & $\begin{array}{l}\text { Cetotifeno } \\
0,05 \%\end{array}$ & $\begin{array}{c}\text { Olopatadina } \\
0,1 \%\end{array}$ & \\
\hline \multirow{5}{*}{ 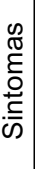 } & Prurido & 4,28 & 3,56 & $-0,614$ \\
\hline & Ardor & 1,89 & 2,06 & $-0,350$ \\
\hline & Lacrimejamento & 1,56 & 1,62 & 0,000 \\
\hline & Secreção & 0,61 & 0,56 & $-0,143$ \\
\hline & Fotofobia & 1,44 & 0,50 & $-1,396$ \\
\hline \multirow{9}{*}{$\frac{\infty}{\pi}$} & Hiperemia & 2,72 & 2,00 & $-0,722$ \\
\hline & Edema pálpebra & 0,77 & 0,25 & $-1,123$ \\
\hline & Edema conjuntiva & 0,83 & 0,69 & $-0,244$ \\
\hline & Cicatrizes & 0,61 & 0,87 & $-0,123$ \\
\hline & Neovascularização & 0,94 & 1,06 & $-0,078$ \\
\hline & Trantas & 1,56 & 1,31 & $-0,104$ \\
\hline & $\begin{array}{l}\text { Epitélio conjuntival corado } \\
\text { com fluoresceína }\end{array}$ & 1,55 & 1,50 & $-0,387$ \\
\hline & Ceratite difusa & 0,94 & 0,12 & $-0,631$ \\
\hline & Ceratite em fenda & 4,17 & 3,00 & $-0,775$ \\
\hline
\end{tabular}

cientes que usaram olopatadina a $0,1 \%, 14$ eram portadores de quadro clínico alérgico leve e 2 com quadro moderado.

A observação das alterações de lubrificação interpretadas pelo teste do tempo de ruptura do filme lacrimal, teste de Schirmer e alterações na integridade do epitélio e os testes laboratoriais de citologia evidenciam também por quê os dois grupos foram homogêneos.

A análise pelo teste de Friedman da evolução da gravidade dos sintomas, sinais e efeitos colaterais nos pacientes tratados com cetotifeno ou olopatadina durante o período estudado evidencia que os sintomas de prurido, ardor e lacrimejamento foram equivalentes e significantemente de menor intensidade. Evidencia-se uma tendência de melhora de fotofobia e secreção na mucosa conjuntival (Tabela 2).

$\mathrm{Na}$ avaliação da evolução do sintoma de prurido, observou-se que o uso do cetotifeno proporcionou melhora significante deste sintoma já a partir do $1^{\circ}$ dia sendo que entre o $7^{\circ}$ e $30^{\circ}$ dia houve a melhora mais significante. O uso da olopatadina também foi eficiente de forma significante em proporcionar a melhora do sintoma prurido, porém não pudemos detectar pelo teste utilizado (TCM) o período em que a melhora foi mais intensa (Tabela 2).

$\mathrm{Na}$ avaliação do sintoma ardor observou-se que o uso do cetotifeno levou a melhora significante, não sendo detectado o período de melhora mais intensa. O uso da olopatadina proporcionou uma diminuição significante do ardor, sendo o período de melhora mais intenso o entre a avaliação feita no $1^{\circ}$ dia de tratamento (Tabela 2).

Realizando-se os testes de associação entre as variáveis para avaliar e comparar o controle dos sintomas prurido, ardor, lacrimejamento, secreção e fotofobia evidencia-se a eqüivalência dos dois tipos de drogas usadas para tratamento de conjuntivite alérgica perene com cetotifeno e a olopatadina. Observou-se uma exceção que ocorreu após 7 dias de tratamento quando os pacientes em uso da olopatadina tinham menos ardor, em relação aos pacientes que fizeram uso do cetotifeno $(\mathrm{p}=0,0305 *$ ou $3,05 \% *)$ (Tabela 3$)$.

Independente do grupo analisado e da intensidade da melhora todos os pacientes do estudo relataram diminuição em seus sintomas com o uso dos colírios.

Quanto aos sinais de hiperemia da conjuntiva bulbar e a integridade do epitélio conjuntival após o uso das medicações tópicas apresentaram melhora significante com ambos os tratamentos. Os demais sinais apresentaram tendência de melhora, sem significância estatística (Tabela 2).

$\mathrm{O}$ uso tópico do cetotifeno foi eficaz em propiciar a tendência de melhora da hiperemia conjuntival, já a partir do $1^{\circ}$ dia e com melhora significante no $14^{\circ} \mathrm{dia}$. A intensidade desta ação farmacológica foi estável entre $1^{\circ}$ ao $30^{\circ}$ dia. Foi observado também melhora significante da hiperemia nos pacientes que usaram a olopatadina tópica, porém alguns pacientes tiveram tendência de apresentar novamente hiperemia conjuntival no $30^{\circ}$ dia (Tabela 2 ).

Para os sinais de: edema de pálpebra; edema de conjun- 


\begin{tabular}{|c|c|c|c|c|c|c|c|c|c|c|}
\hline \multicolumn{2}{|r|}{ Varíaveis analisadas $^{\dagger}$} & Colírios & Dia pré & $1^{\circ} \mathrm{dia}$ & $2^{\circ} \mathrm{dia}$ & $7^{\circ} \mathrm{dia}$ & $14^{\circ}$ dia & $30^{\circ} \mathrm{dia}$ & $\begin{array}{l}\text { Teste de } \\
\text { Friedman } \chi^{2}\end{array}$ & $\begin{array}{l}\text { Teste das comparações } \\
\text { múltiplas }\end{array}$ \\
\hline \multirow{5}{*}{ 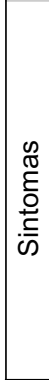 } & Prurido & $\begin{array}{l}\text { Cetotifeno } \\
\text { Olopatadina }\end{array}$ & $\begin{array}{l}1,333 \\
1,000\end{array}$ & $\begin{array}{l}0,611 \\
0,438\end{array}$ & $\begin{array}{l}0,722 \\
0,438\end{array}$ & $\begin{array}{l}0,500 \\
0,563\end{array}$ & $\begin{array}{l}0,556 \\
0,500\end{array}$ & $\begin{array}{l}0,556 \\
0,625\end{array}$ & $\begin{array}{c}18,570^{*} \\
13,226\end{array}$ & $\begin{array}{l}{ }^{*} \text { Pré }>7^{\circ} \text { dia, pré }>14^{\circ} \text { dia, pré }>30^{\circ} \text { dia } \\
\text { *O teste não conseguiu detectar diferença }\end{array}$ \\
\hline & Ardor & $\begin{array}{l}\text { Cetotifeno } \\
\text { Olopatadina }\end{array}$ & $\begin{array}{l}0,722 \\
0,813\end{array}$ & $\begin{array}{l}0,278 \\
0,500\end{array}$ & $\begin{array}{l}0,111 \\
0,188\end{array}$ & $\begin{array}{l}0,389 \\
0,062\end{array}$ & $\begin{array}{l}0,111 \\
0,188\end{array}$ & $\begin{array}{l}0,278 \\
0,313\end{array}$ & $\begin{array}{l}19,305^{\star} \\
24,719^{\star}\end{array}$ & $\begin{array}{l}\text { O teste não conseguiu detectar diferença } \\
\text { Pré }>7^{\circ} \text { dia }\end{array}$ \\
\hline & Lacrimejamento & $\begin{array}{l}\text { Cetotifeno } \\
\text { Olopatadina }\end{array}$ & $\begin{array}{l}0,611 \\
0,688\end{array}$ & $\begin{array}{l}0,222 \\
0,250\end{array}$ & $\begin{array}{l}0,278 \\
0,125\end{array}$ & $\begin{array}{l}0,111 \\
0,188\end{array}$ & $\begin{array}{l}0,111 \\
0,125\end{array}$ & $\begin{array}{l}0,222 \\
0,250\end{array}$ & $\begin{array}{l}16,203^{*} \\
22,778^{*}\end{array}$ & $\begin{array}{l}\text { O teste não conseguiu detectar diferença } \\
\text { O teste não conseguiu detectar diferença }\end{array}$ \\
\hline & Secreção & $\begin{array}{l}\text { Cetotifeno } \\
\text { Olopatadina }\end{array}$ & $\begin{array}{l}0,222 \\
0,188\end{array}$ & $\begin{array}{l}0,111 \\
0,125\end{array}$ & $\begin{array}{l}0,111 \\
0,062\end{array}$ & $\begin{array}{l}0,055 \\
0,000\end{array}$ & $\begin{array}{l}0,055 \\
0,062\end{array}$ & $\begin{array}{l}0,055 \\
0,125\end{array}$ & $\begin{array}{l}5,541 \\
5,000\end{array}$ & $\begin{array}{l}- \\
-\end{array}$ \\
\hline & Fotofobia & $\begin{array}{l}\text { Cetotifeno } \\
\text { Olopatadina }\end{array}$ & $\begin{array}{l}0,333 \\
0,250\end{array}$ & $\begin{array}{l}0,389 \\
0,062\end{array}$ & $\begin{array}{l}0,278 \\
0,062\end{array}$ & $\begin{array}{l}0,111 \\
0,062 \\
\end{array}$ & $\begin{array}{l}0,055 \\
0,000\end{array}$ & $\begin{array}{l}0,278 \\
0,062\end{array}$ & $\begin{array}{l}10,077 \\
10,769\end{array}$ & $\begin{array}{l}- \\
-\end{array}$ \\
\hline \multirow{9}{*}{$\begin{array}{l}\frac{\infty}{\pi} \\
\frac{5}{\omega}\end{array}$} & Hiperemia & $\begin{array}{l}\text { Cetotifeno } \\
\text { Olopatadina }\end{array}$ & $\begin{array}{l}0,889 \\
0,065\end{array}$ & $\begin{array}{l}0,500 \\
0,250\end{array}$ & $\begin{array}{l}0,500 \\
0,250\end{array}$ & $\begin{array}{l}0,333 \\
0,250\end{array}$ & $\begin{array}{l}0,167 \\
0,125\end{array}$ & $\begin{array}{l}0,333 \\
0,500\end{array}$ & $\begin{array}{l}27,255^{\star} \\
17,895^{\star}\end{array}$ & $\begin{array}{l}\text { Pré }>14^{\circ} \text { Dia } \\
\text { O teste não conseguiu detectar diferença }\end{array}$ \\
\hline & Edema de pálpebra & $\begin{array}{l}\text { Cetotifeno } \\
\text { Olopatadina }\end{array}$ & $\begin{array}{l}0,278 \\
0,188\end{array}$ & $\begin{array}{l}0,167 \\
0,000\end{array}$ & $\begin{array}{l}0,111 \\
0,000\end{array}$ & $\begin{array}{l}0,055 \\
0,062\end{array}$ & $\begin{array}{l}0,111 \\
0,000\end{array}$ & $\begin{array}{l}0,055 \\
0,000\end{array}$ & $\begin{array}{l}7,593 \\
8,750\end{array}$ & - \\
\hline & $\begin{array}{l}\text { Edema de } \\
\text { conjuntiva bulbar }\end{array}$ & $\begin{array}{l}\text { Cetotifeno } \\
\text { Olopatadina }\end{array}$ & $\begin{array}{l}0,222 \\
0,125\end{array}$ & $\begin{array}{l}0,222 \\
0,188\end{array}$ & $\begin{array}{l}0,167 \\
0,062\end{array}$ & $\begin{array}{l}0,111 \\
0,125\end{array}$ & $\begin{array}{l}0,055 \\
0,062\end{array}$ & $\begin{array}{l}0,055 \\
0,125\end{array}$ & $\begin{array}{l}8,571 \\
6,538\end{array}$ & $\begin{array}{l}- \\
-\end{array}$ \\
\hline & Cicatrizes & $\begin{array}{l}\text { Cetotifeno } \\
\text { Olopatadina }\end{array}$ & $\begin{array}{l}0,111 \\
0,188\end{array}$ & $\begin{array}{l}0,055 \\
0,188\end{array}$ & $\begin{array}{l}0,111 \\
0,125\end{array}$ & $\begin{array}{l}0,111 \\
0,125\end{array}$ & $\begin{array}{l}0,111 \\
0,125\end{array}$ & $\begin{array}{l}0,111 \\
0,125\end{array}$ & $\begin{array}{l}5,000 \\
4,000\end{array}$ & $\begin{array}{l}- \\
-\end{array}$ \\
\hline & Neovasscularização & $\begin{array}{l}\text { Cetotifeno } \\
\text { Olopatadina }\end{array}$ & $\begin{array}{l}0,167 \\
0,250\end{array}$ & $\begin{array}{l}0,111 \\
0,250\end{array}$ & $\begin{array}{l}0,167 \\
0,125\end{array}$ & $\begin{array}{l}0,167 \\
0,188\end{array}$ & $\begin{array}{l}0,167 \\
0,125\end{array}$ & $\begin{array}{l}0,167 \\
0,125\end{array}$ & $\begin{array}{l}5,000 \\
7,632\end{array}$ & $\begin{array}{l}- \\
-\end{array}$ \\
\hline & Nódulos de Trantas & $\begin{array}{l}\text { Cetotifeno } \\
\text { Olopatadina }\end{array}$ & $\begin{array}{l}0,278 \\
0,313\end{array}$ & $\begin{array}{l}0,278 \\
0,250\end{array}$ & $\begin{array}{l}0,222 \\
0,188\end{array}$ & $\begin{array}{l}0,222 \\
0,188\end{array}$ & $\begin{array}{l}0,222 \\
0,188\end{array}$ & $\begin{array}{l}0,333 \\
0,188\end{array}$ & $\begin{array}{l}3,696 \\
4,000\end{array}$ & $\begin{array}{l}- \\
-\end{array}$ \\
\hline & $\begin{array}{l}\text { Epitélio conjuntival co- } \\
\text { rado com fluoresceina }\end{array}$ & $\begin{array}{l}\text { Cetotifeno } \\
\text { Olopatadina }\end{array}$ & $\begin{array}{l}0,333 \\
0,250\end{array}$ & $\begin{array}{l}0,444 \\
0,438\end{array}$ & $\begin{array}{l}0,278 \\
0,313\end{array}$ & $\begin{array}{l}0,167 \\
0,250\end{array}$ & $\begin{array}{l}0,278 \\
0,125\end{array}$ & $\begin{array}{l}0,055 \\
0,125\end{array}$ & $\begin{array}{l}12,174^{*} \\
12,273^{*}\end{array}$ & $\begin{array}{l}\text { O teste não conseguiu detectar diferença } \\
\text { O teste não conseguiu detectar diferença }\end{array}$ \\
\hline & Ceratite difusa & $\begin{array}{l}\text { Cetotifeno } \\
\text { Olopatadina }\end{array}$ & $\begin{array}{l}0,167 \\
0,000\end{array}$ & $\begin{array}{l}0,167 \\
0,000\end{array}$ & $\begin{array}{l}0,167 \\
0,062\end{array}$ & $\begin{array}{l}0,167 \\
0,000\end{array}$ & $\begin{array}{l}0,167 \\
0,000\end{array}$ & $\begin{array}{l}0,111 \\
0,062\end{array}$ & $\begin{array}{l}5,000 \\
4,000\end{array}$ & $\begin{array}{l}- \\
- \\
-\end{array}$ \\
\hline & Ceratite em fenda & $\begin{array}{l}\text { Cetotifeno } \\
\text { Olopatadina }\end{array}$ & $\begin{array}{l}0,667 \\
0,438\end{array}$ & $\begin{array}{l}0,778 \\
0,438\end{array}$ & $\begin{array}{l}0,722 \\
0,438\end{array}$ & $\begin{array}{l}0,667 \\
0,625\end{array}$ & $\begin{array}{l}0,722 \\
0,500\end{array}$ & $\begin{array}{l}0,611 \\
0,563\end{array}$ & $\begin{array}{l}3,000 \\
1,542\end{array}$ & - \\
\hline
\end{tabular}

tiva; cicatrizes em tarso superior; neovascularização; nódulos de "Trantas"; ceratite difusa e ceratite em fenda não houve diferença significante tanto no grupo dos pacientes tratados com cetotifeno como com a olopatadina (Tabela 2).

A avaliação e comparação de melhora dos sinais de conjuntivite alérgica realizados pelos testes de associação entre as variáveis evidenciaram uma eqüivalência das respostas observadas com os tratamentos estudados, com uma exceção no $2^{\circ}$ dia de tratamento onde os pacientes que usaram a olopatadina apresentaram menos ceratite em fenda em relação aos pacientes que usaram cetotifeno.

Os sinais de papilas em conjuntiva tarsal superior e a intensidade de edema no limbo corneal que evoluíram com melhora após o tratamento, são de difícil mensuração e optamos por não aplicar a análise estatística.

As únicas reações adversas relatadas após o uso dos dois colírios foram: prurido e ardor. Os pacientes que usaram cetotifeno apresentaram prurido e ardor significantes como reação adversa, não sendo possível pelo teste de comparação múltipla detectar em que período esta reação foi pior. Em relação à análise do uso de olopatadina levando a reações adversas verificou-se que o ardor foi significante para o grupo estudado, não sendo possível detectar o período de piora desta reação (Tabela 4). Os resultados da avaliação das reações adversas não evidenciaram diferença significante entre os tratamentos efetuados nas tabelas de associação.

$\mathrm{O}$ tratamento da conjuntivite alérgica com cetotifeno ou olopatadina modificou, mas de forma não significante, o tempo de ruptura do filme lacrimal e os resultados do teste de Schirmer. Os mesmos resultados analisados comparando-se os efeitos de um e outro tratamento nestas variáveis evidenciam a equivalência dos tratamentos. No exame citológico da conjuntiva realizado antes do tratamento observa-se neutrófilos degenerados, eosinófilos íntegros e degenerados, presença de grânulos livres. Com ambos os tratamentos observase alteração do número de grânulos eosinofílicos detectados, porém esta tendência não é estatisticamente significante. A comparação dos resultados observados entre os grupos evidencia a equivalência entre os tratamentos. A análise do número de culturas positivas da conjuntiva em cada grupo antes 


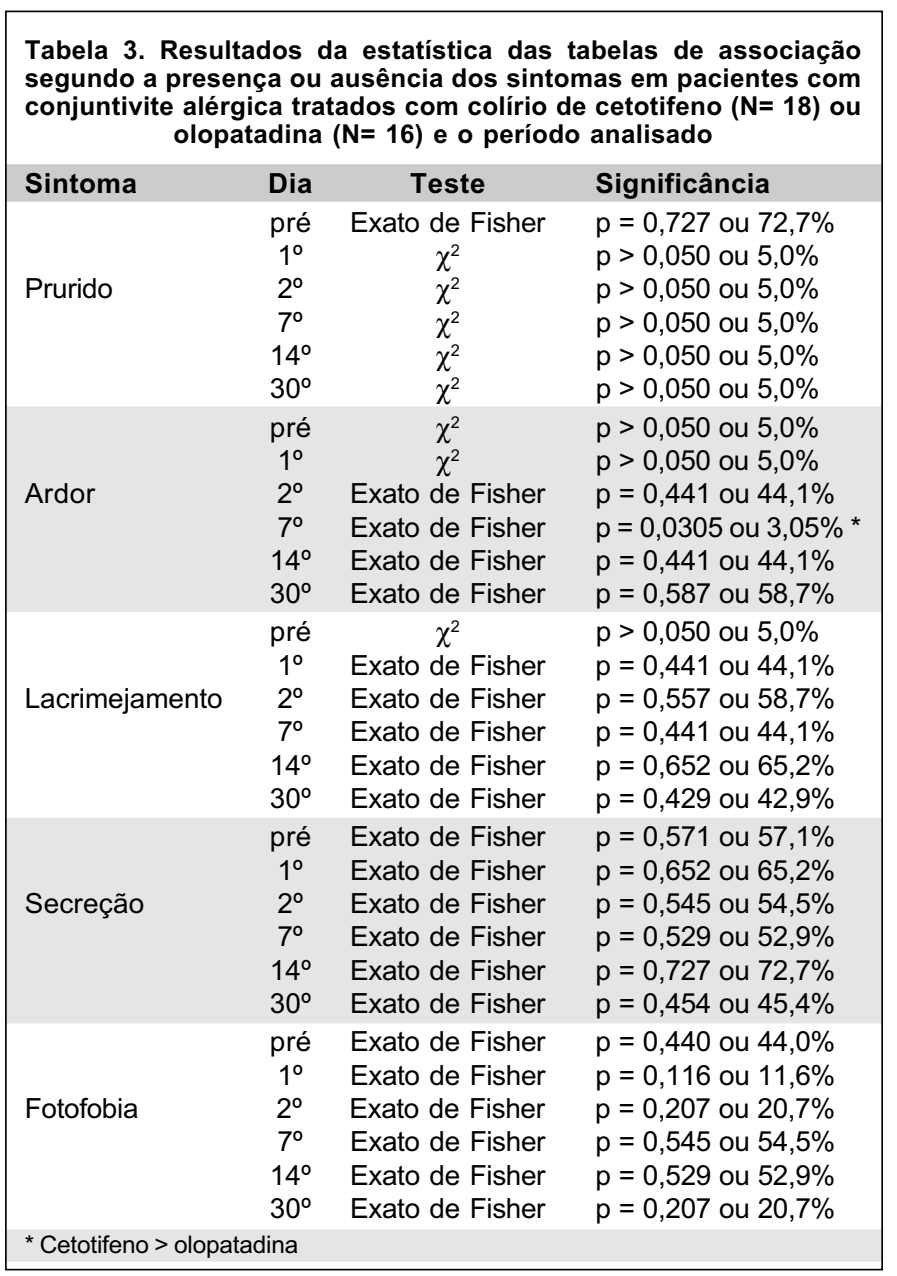

e depois do tratamento evidencia que o tratamento não mudou de forma significante a microbiota da conjuntiva.

DISCUSSÃO

Foi possível pela comparação entre os grupos tratados em relação à gravidade dos sinais e sintomas de alergia ocular demonstrar que os grupos eram homogêneos, permitindo a análise deste estudo.

A aceitação dos pacientes às drogas do estudo foi boa, não houve desistência do tratamento devido intolerância ou efeitos colaterais. Resultados semelhantes foram observados por outros autores em relação a olopatadina tópica ${ }^{(15)}$ e ao cetotifeno tópico ${ }^{(16-17)}$.

Optamos por não usar colírio placebo nos pacientes com alergia para a análise comparativa, pois o placebo pode diminuir a manifestação alérgica pela ação mecânica de diluição dos antígenos e gerar resultados falso-positivos nas respostas ao tratamento ${ }^{(18)}$.

As soluções oftálmicas de cetotifeno e olopatadina como droga antialérgica de uso tópico foram testadas e significantemente eficazes na diminuição de sintomas e sinais importantes da doença alérgica ocular como o prurido, o ardor e o lacrimejamento, o que também foi observado por estudos anteriores $^{(15,17)}$.

A hiperemia conjuntival foi também avaliada e representa importante parâmetro para os pacientes quanto à melhora da doença. Verificou-se com o uso dos colírios melhora significante na hiperemia conjuntival, concordando com estudos

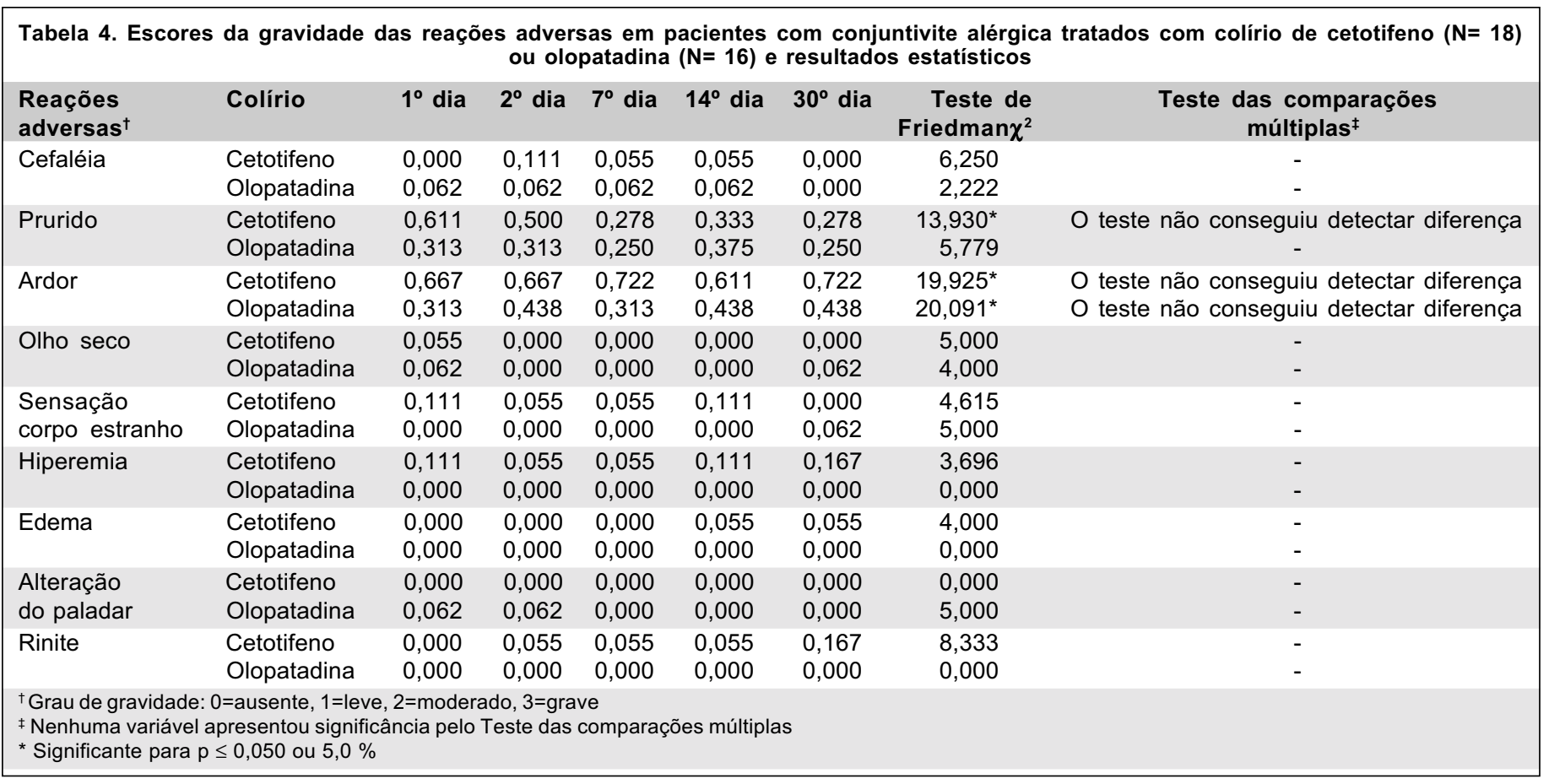


anteriores que estudaram pacientes alérgicos tratados com olopatadina a $0,05 \%{ }^{(15)}$. Relatos sobre regressão da hiperemia conjuntival com a instilação de cetotifeno tópico são escassos na literatura.

Como observado em outros estudos a instilação dos colírios de olopatadina e cetotifeno a $0,05 \%$ a cada 12 horas, foi suficiente para provocar o efeito farmacológico desejado ${ }^{(15)}$. A instilação duas vezes por dia é um fator importante em facilitar a manutenção dos pacientes em tratamento, principalmente em casos de doença alérgica da conjuntiva de caráter sazonal ou perene, onde algumas medicações são instiladas mais freqüentemente para garantir o seu efeito terapêutico. Algumas drogas antialérgicas preconizadas para instilação de três ou quatro vezes ao dia, são a lodoxamida, levocabastina e o ketorolac ${ }^{(19)}$. A comparação das soluções oftálmicas de cetotifeno a $0,025 \%$ e $0,05 \%$ instiladas quatro vezes ao dia em pacientes com conjuntivite alérgica apresenta resultados de melhora dos sintomas entre os pacientes que instilaram o cetotifeno a $0,025 \%$ em $23 \%$ dos casos, $85 \%$ dos pacientes que instilaram solução oftálmica de cetotifeno a $0,05 \%$ obtiveram melhora. Com estas observações concluiu-se que a solução de cetotifeno a $0,025 \%$ não foi eficaz na redução dos sintomas da alergia ocular nos pacientes avaliados como a solução de cetotifeno a $0,05 \%^{(17)}$, dados estes semelhantes aos observados neste estudo.

Entre os pacientes estudados observou-se o ardor como reação adversa tanto em pacientes usando cetotifeno como olopatadina, ocorrendo durante os 20 a 30 minutos seguintes à instilação. Os pacientes que usaram cetotifeno relataram também prurido após a instilação, sintoma este que diminuiu de intensidade no transcorrer do tratamento. Estas queixas podem ser justificadas por incompatibilidade de $\mathrm{pH}$ entre a solução oftálmica e a conjuntiva ${ }^{(20)}$, pois o uso tópico destes medicamentos não levou a nenhuma reação de hipersensibilidade local ou sistêmica.

O cetotifeno como uma nova droga antialérgica tópica, ao ser comparada a olopatadina tópica, teve seus efeitos de controle dos sintomas e sinais das conjuntivites alérgicas eficazes e estáveis durante os 30 dias do estudo. Apresentando um uso seguro e bem tolerado na posologia de duas vezes por dia, com intervalo médio de 12 horas. Todos pacientes consideraram seu uso confortável, semelhante aos resultados obtidos com a olopatadina tópica.

Concluímos que ambas são drogas equivalentes e atuaram de forma eficaz e segura na remissão dos sintomas relacionados à conjuntivite alérgica.

\section{A B S T RAC T}

Purpose: To evaluate and compare the efficacy and tolerance to the topical use of $0.05 \%$ ketotifen fumarate and $0.1 \%$ olopatadine hydrochloride in the treatment of patients with allergic conjunctivitis. Methods: A masked, randomized clinical study was performed in order to compare the efficacy, safety and side effects of the use of $0.05 \%$ ketotifen fumarate and $0.1 \%$ olopatadine hydrochloride ophthalmic solutions for the alleviation of symptoms and signs in patients with allergic conjunctivitis. Thirty-four patients, fulfilling the inclusion criteria of the protocol were divided into two groups and received a flask with the masked drug, instilling one drop twice daily in each eye for 30 days. Signs and symptoms of these patients were evaluated on a visit before treatment and on five visits during the treatment (days 1, 2, 7, 14 and 30). Results: Severity of allergic conjunctivitis was the same in both studied groups. Both ketotifen and olopatadine were equivalent and efficient regarding decrease in itching, burning and lacrimation symptoms. Bulbar conjunctival hyperemia was attenuated in both groups. Evaluation of adverse reactions showed the occurrence of burning on administration of both drugs and ketotifen led to occurrence of itching. No hypersensitivity reaction to the studied drugs was observed. Conclusions: This study evidences that $0.05 \%$ ketotifen fumarate and $0.1 \%$ olopatadine hydrochloride ophthalmic solutions, when instilled twice daily for 30 days, were efficient and safe regarding alleviation of the main symptoms and signs of allergic conjunctivitis.

Keywords: Ketotifen/therapeutic use; Dibenzoxepins/therapeutic use; Ophthalmic solutions; Conjunctivitis, allergic/ drug therapy

\section{REFERENCIAS}

1. Sheldrick JH, Vernon SA, Wilson A, Read SJ. Demand incidence and episode rates of ophthalmic disease in a defined urban population. BMJ 1992; 305:933-6.

2. Brody JM, Foster CS. Vernal conjunctivitis. In: Pepose JS, Holland GN, Wilhelmus KR, editors. Ocular infection \& immunity. St Louis: Mosby; 1996. p. 367-75

3. Liesegang TJ. Atopic keratoconjunctivitis. In: Pepose JS, Holland GN, Wilhelmus KR, editors. Ocular infection \& immunity. St Louis: Mosby; 1996. p. 376-90.

4. Reiss J, Abelson MB, George MA, Wedner HJ. Allergic conjunctivitis. In: Pepose JS, Holland GN, Wilhelmus KR, editors. Ocular infection \& immunity. St Louis: Mosby; 1996. p. 345-58.

5. McGill JI, Holgate ST, Church MK, Anderson DF, Bacon A. Allergic eye disease mechanisms. Br J Ophthalmol 1998;82:1203-14.

6. Abelson MB, Madiwale N, Weston JH. Conjunctival eosinophils in allergic ocular disease. Arch Ophthalmol 1983;101:555-6.

7. Friedlaender MH, Okumoto Y, Kelley J. Diagnosis of allergic conjunctivitis. Arch Ophthalmol 1984;102:1198-9.

8. Sharif NA, Xu SX, Miller ST, Yanni JM. Olopatadine (AL-4943A): ligand binding and functional studies on a novel, long acting H1-selective histamine antagonist and anti-allergic agent for use in allergic conjunctivitis. J Ocul Pharmacol Ther 1996;12:401-7.

9. Carrasco E, Galleguillos F, Bernath Z. The orally administered anti-allergic agent, ketotifen; efficacy in atopic and non-atopic bronchial asthma. Allergol Immunopathol (Madr)1981;9:335-42.

10. Craps L. Ketotifen in the oral prophylaxis of bronchial asthma: a review. Pharmatherapeutica 1981;3:18-35.

11. Heyman SN, Karmeli F, Brezis M, Rachimilewitz D. The effect of ketotifen on nitric oxide synthase activity. Br J Pharmacol 1997;120:1545-51.

12. Grant SM, Goa KL, Fitton A, Sorkin EM. Ketotifen. A review of its pharmacodynamic and pharmacokinetic properties, and therapeutic use in asthma and allergic disorders. Drugs 1990;40:412-48.

13. Holander M, Wolfe DA. Non-parametric statistical methods. New York: John Wiley \& Sons; 1974. 
14. Siegel S. Non-parametric statistics for the behavioral sciences. New York: Mc Graw-Hill; 1956

15. Abelson MB, Spitalny L. Combined analysis of two studies using the conjunctival allergen challenge model to evaluate olopatadine hydrochloride, a new ophthalmic antiallergic agent with dual activity. Am J Ophthalmol 1998; 125:797-804.

16. Mikuni I, Nagajima A, Kogure F, Uchida Y, Kitano S, Shimizu Y, Yuasa T, Mimura Y. Evaluation of ketotifen ophthalmic solution on efficacy and safety on allergy conjunctivitis and vernal conjunctivitis: results on multiclinic open trial. Rinsho Iyaku 1988;4:2371-83.

17. Mikuni I, Nagajima A, Kogure F, Uchida Y, Kitano S, Shimizu Y, Yuasa T,
Mimura Y. Clinical effect of ketotifen ophthalmic solution on allergic conjunctivitis and vernal conjunctivitis: multicentre double-blind study in comparisons with disodium cromoglycate ophthalmic solution. Rinsho Hyoka 1989;17:275-9.

18. Abelson MB, Chambers WA, Smith LM. Conjunctival allergen challenge. Arch Ophthalmol 1990;108:84-8.

19. Verin P. Treating severe eye allergy. Clin Exp Allergy 1998;28 Suppl 6:44-8.

20. Bar-Ilan A, Nuemann R. Basic considerations of ocular drug-delivery systems. In: Zimmerman TJ, Kooner KS, Sharir M, Fechtner RD, editors. Textbook of ocular pharmacology. Philadelphia: Lippincott-Raven; 1997. p. 139-55.

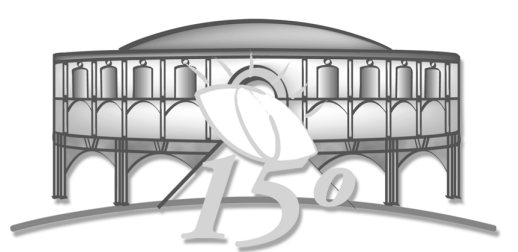

Congresso Brasileiro de Prevenção da Cegueira e Reabilitação Visual

\section{CONSELHO BRASILEIRO} DE OFTALMOLOGIA

\section{CONGRESSO BRASILEIRO DE}

\section{PREVENÇÃO DA CEGUEIRA E REABILITAÇÃO VISUAL}

\section{A 4 DE SETEMBRO DE 2002 \\ EXPO TRADE - CURITIBA - PR}

Comissão Executiva:

\author{
Dra. Saly Moreira \\ Dr. Jayme Arana \\ Dr. Kenji Sakata \\ Dr. Hamilton Moreira \\ Dr. José Joaquim Júnior \\ Dr. Ivo Luvizotti \\ Dr. José Jorge Neto
}

\author{
Presidente \\ $1^{\circ}$ Vice-Presidente \\ $2^{\circ}$ Vice-Presidente \\ $1 \circ$ Secretário \\ $2 \circ$ Secretário \\ $1 \circ$ Tesoureiro \\ $2^{\circ}$ Tesoureiro
}

Secretaria Executiva: $\quad C B O$ Eventos

Al. Santos, 1343 - Conj. 505 - CEP: 01419-001 - São Paulo - SP

Tel: (1 1) 32664000 / Fax: (1 1) 31710948 - Email: eventos@cbo.com.br 